\title{
Nonrelativistic Atomic Spectrum for Companied Harmonic Oscillator Potential and its Inverse in Both NC-2D: RSP
}

\author{
Abdelmadjid Maireche \\ Physics Department, Sciences faculty, University of M'sila, Algeria \\ Corresponding Author, E-mails: abmaireche@yahoo.fr and abmaireche@gmail.com
}

\begin{abstract}
Keywords: the companied Harmonic oscillator potential and its inverse, the isotropic harmonic oscillator plus inverse quadratic potential, Noncommutative space and phase, Boopp's shift method, Boopp's shift method.
\end{abstract}

\begin{abstract}
A novel study for the exact solvability of nonrelativistic quantum spectrum systems for companied Harmonic oscillator potential and its inverse (the isotropic harmonic oscillator plus inverse quadratic potential) is discussed used both Boopp's shift method and standard perturbation theory in both noncommutativity two dimensional real space and phase (NC-2D: RSP), furthermore the exact corrections for the spectrum of studied potential was depended on two infinitesimals parameters $\theta$ and $\bar{\theta}$ which plays an opposite rolls, this permits us to introduce a new fixing gauge condition and we have also found the corresponding noncommutative anisotropic Hamiltonian.
\end{abstract}

\section{INTRODUCTION}

One of the important problems in relativistic and nonrelativistic quantum mechanics is to find exact solutions to the Dirac, Klein-Gordon and Schrödinger equations for spherical and non spherical potentials that are used in different fields of physics and materials sciences, in commutative and noncommutative spaces-phases at two and three dimensional spaces [1-37]. In particular, the fermionic particle with spin $(1 / 2)$ interacted with proton and in other hand interacted with external field produced by Harmonic oscillator and its inverse under investigation [7]. The application of noncommutativity properties on physics fields satisfied by imposing one or two new commutators $(c=\hbar=1)[19-37]$ :

$$
\left[x_{i}, x_{j}\right]_{*}=i \theta_{i j} \text { and }\left[\hat{p}_{i}, \hat{p}_{j}\right]_{*}=i \bar{\theta}_{i j}
$$

It's important to notice that, the above two fundamental commutators are satisfied in particulars' cases from the general star product between two arbitrary functions $f(x)$ and $g(x)$ in the first order of two parameters $\theta$ and $\bar{\theta}_{i j}$ as follow [19-37]:

$$
f(x) * g(x)=f(x) g(x)-\frac{i}{2} \theta^{\mu v}\left(\partial_{\mu}^{x} f(x)\right)\left(\partial_{\nu}^{p} g(x)\right)-\frac{i}{2} \theta^{\mu v}\left(\partial_{\mu}^{p} f(x)\right)\left(\partial_{v}^{p} g(x)\right)
$$

The two parts are represent the effects of the noncommutativity of space and phase, respectively, the parameters $\theta^{i j}$ and $\bar{\theta}_{i j}$ are antisymmetric real matrixes. At (NC- $N$ D: RSP), a Boopp's shift method can be used, instead of solving any quantum systems by using directly star product procedure:

$$
\left[\hat{x}_{i}, \hat{x}_{j}\right]=i \theta_{i j} \text { and }\left[\hat{p}_{i}, \hat{p}_{j}\right]=i \bar{\theta}_{i j}
$$

In present work, the star product replaced by usual product together with a Boopp's shift in (NC-2D: RSP) [26-35]:

$$
\left\{\begin{array}{l}
\hat{x}=x-\frac{\theta}{2} p_{y}, \quad \hat{y}=y+\frac{\theta}{2} p_{x} \\
\hat{p}_{x}=p_{x}+\frac{\bar{\theta}}{2} y \text { and } \quad \hat{p}_{y}=p_{x}-\frac{\bar{\theta}}{2} x
\end{array}\right.
$$

We can prove, that: $[\hat{x}, \hat{y}],\left\lfloor\hat{p}_{x}, \hat{p}_{y}\right\rfloor, \Delta x \Delta y$ and $\Delta p_{x} \Delta p_{y}$ are only non null two commutators and the two uncertainties, respectively: 


$$
\left\{\begin{array}{l}
{[\hat{x}, \hat{y}]=i \theta, \quad\left[\hat{p}_{x}, \hat{p}_{y}\right]=-i \bar{\theta}} \\
\Delta x \Delta y \approx \theta \text { and } \quad \Delta p_{x} \Delta p_{y} \approx \bar{\theta}
\end{array}\right.
$$

Here $\theta \equiv \theta^{12}$ and $\bar{\theta} \equiv \bar{\theta}^{12}$, its worth to mention, that the above two uncertainties, can be deduced from the generalized incertitude relations to the special coordinates and impulsions in noncommutative $N$ dimensional space and phase, respectively:

$$
\Delta x^{i} \Delta x^{j} \geq \frac{1}{2}\left|\theta^{i j}\right|
$$

And

$$
\Delta p^{i} \Delta p^{j} \geq \frac{1}{2}\left|\bar{\theta}^{i j}\right|
$$

The main aim of this paper is to present and study a companied Harmonic potential and its inverse (h.p.i) in (NC-2D RSP) to discover the new symmetries and a possibility to obtain another application to this potential in different fields. The rest of present search is organized as follows: In next section, we briefly review the Schrödinger equation with companied Harmonic potential and it's inverse. The Section 3, reserved to derive the deformed Hamiltonians of the Schrödinger equation with companied (h.p.i) and by applying both Boopp's shift method and standard perturbation theory we find the quantum spectrum of the lowest excitations in (NC-2D RSP) for studied potential. Finally, the important found results and the conclusions are discussed in the four and last section.

\section{REVIEW OF COMPAINED HARMONIC POTANTIAL AND ITS INVERSE IN ORDINARY TWO DIMENSIONAL SPACES}

Let's present a brief review of time independent Schrödinger equation for a fermionic particle like electron of rest mass $m_{0}$ and its energy $E$ moving in companied (h.p.i) [7]:

$$
\left(-\frac{1}{2 m_{0}}\left(\frac{1}{r} \frac{\partial}{\partial r}\left(r \frac{\partial}{\partial r}\right)+\frac{1}{r^{2}} \frac{\partial^{2}}{\partial \varphi^{2}}\right)+a r^{2}+\frac{b}{r^{2}}\right) \Psi(\dot{*} r)=E \Psi(\dot{r})
$$

Where $\left(a=\frac{1}{2} m_{0} \omega^{2}, b\right)$ are both constants. In polar coordinates, the complete wave function $\Psi\left(\begin{array}{l}r \\ r\end{array}\right)$ separated as follows:

$$
\Psi(\ddot{r})=R_{n l}(r) \Phi(\varphi)
$$

Where the radial function $R_{n l}(r)$ and the angular function $\Phi(\varphi)$ are satisfied the following two equations, in 2D space respectively [7]:

$$
\begin{gathered}
\left(\frac{d^{2}}{d r^{2}}+\frac{1}{r} \frac{d}{d r}-\frac{m^{2}}{r^{2}}-2 m_{0}(E-V(r))\right) R_{n l}(r)=0 \\
\frac{d^{2} \Phi_{m}(\varphi)}{d \varphi^{2}}+m^{2} \Phi_{m}(\varphi)=0
\end{gathered}
$$

Here $m$ denote to the orbital angular momentum quantum numbers, the standard solution of $\Phi(\varphi)$ given by:

$$
\Phi(\varphi)=\frac{1}{\sqrt{2 \pi}} \exp ( \pm i m \varphi)
$$

Using the following abbreviations [7]:

$$
v(v+1)=m^{2}+2 m_{0} b, \mu^{2}=2 m_{0} a \text { and }: \varepsilon^{2}=2 m_{0} E
$$

Then, a radial function satisfied the following differential equation [7]:

$$
\left(\frac{d^{2}}{d r^{2}}+\frac{1}{r} \frac{d}{d r}-\frac{v(v+1)}{r^{2}}-\mu^{2} r^{2}+\varepsilon^{2}\right) R_{n l}(r)=0
$$

Winches accept the solution:

$$
R_{n l}(r)=C_{n l} \exp \left(-\frac{\mu}{2} \cdot r^{2}\right) r_{1}^{k} F\left(-n, k+1, r^{2}\right)
$$

Where the function ${ }_{1} F_{1}\left(-n, k+1, r^{2}\right)$ is given by [6]: 


$$
{ }_{1} F_{1}\left(-n, k+1, r^{2}\right)=\frac{n ! k !}{(n+k) !} L_{(n)}^{(k)}\left(r^{2}\right)
$$

And $C_{n l}$ is the normalization constant, determined from the condition [7]:

$$
C_{n l}=\left(\frac{\sqrt{2} \mu^{\frac{1}{2}(k+1)} n !}{\Gamma(k+n+1)}\right)^{\frac{1}{2}} \frac{(n+k) !}{n ! k !}
$$

The complete orthonormalization eignenfunctions and the energy eigenvalues respectively in two dimensional spaces [7]:

$$
\begin{gathered}
\frac{\Psi(r, \varphi)}{\exp ( \pm i m \varphi)}=\frac{\mu^{\frac{1}{2}(k+1)}}{\sqrt{\pi}}\left(\frac{n !}{\Gamma(k+n+1)}\right)^{\frac{1}{2}} \frac{(n+k) !}{n ! k !} \exp \left(-\frac{\mu}{2} r^{2}\right) r^{k}{ }_{1} F\left(-n, k+1, r^{2}\right) \\
E_{n l}=\sqrt{\frac{8 \hbar^{2}}{M}}\left(n+\frac{1}{2}+\frac{1}{4}\left(4 m^{2}+\frac{8 M b}{\hbar^{2}}\right)^{\frac{1}{2}}\right)=\omega\left(2 n+\left(k=m^{2}+2 M b\right)+1\right)
\end{gathered} .
$$

\section{TWO DIMENSIONAL NONCOMMUTATIVE REAL SPACE AND PHASE FOR COMPAINED HARMONIC POTANTIAL AND ITS INVERSE}

In this section, we present some fundamental principles of Schrödinger equation in (NC-2D: RSP); applying the important 4-steps on the ordinary quantum Schrödinger equation [27-36]:

$$
\begin{aligned}
& \text { Ordinary } 2 \mathrm{D}-\text { Hamiltonian }: \hat{H}\left(p_{i}, x_{i}\right) \\
& \rightarrow \mathrm{NC} \quad \text { 2D-Hamiltonian }: \hat{H}\left(\hat{p}_{i}, \hat{x}_{i}\right) \\
& \text { Ordinary complex wave function }: \Psi(\vec{r}) \\
& \rightarrow \mathrm{NC} \quad \text { complex wave function }: \hat{\Psi}(\vec{r}) \\
& \text { Ordinary energy }: E \rightarrow \mathrm{NC} \text { energy }: E_{n c-i h}
\end{aligned}
$$

And

$$
\begin{aligned}
& \text { Ordinary product }: . \rightarrow \\
& \text { New star producr acting on phase and space :* }
\end{aligned}
$$

This implies the Schrödinger equitation in both (NC-2D RSP) as:

$$
\hat{H}\left(\hat{p}_{i}, \hat{x}_{i}\right) * \hat{\Psi}(\vec{r})=E_{n c-i h} \hat{\Psi}(\vec{r})
$$

Now, we apply the Boopp's shift method on the above equation to obtain, the reduced Schrödinger equation (without star products):

$$
H\left(\hat{p}_{i}, \hat{x}_{i}\right) \psi(\vec{r})=E_{n c-i h} \psi(\vec{r})
$$

This is a translation of a Schrödinger equation for $\hat{p}_{i}$ and $\hat{x}_{i}$ with the same complex wave function $\psi(\vec{r})$. As a direct result of the eq. (4), the two operators $\hat{r}^{2}$ and $\hat{p}^{2}$ in (NC-2D RSP) can be written as follows [24-30]

$$
\left\{\begin{array}{l}
\hat{r}^{2}=r^{2}-\theta L_{z} \\
\hat{p}^{2}=p^{2}+\bar{\theta} L_{z} \quad \text { with } L_{z} \equiv x p_{y}-y p_{x}
\end{array}\right.
$$

It's important to notice that, the two obtained results in eq. (21) conserved the symmetry between $\hat{p}_{i}$ and $\hat{x}_{i}$ and the rolls of $\theta$ and $\bar{\theta}$ are inversed $(\theta \approx-\bar{\theta})$. Based, on the eq. (21), to obtain, after a straightforward calculation, the three important terms, which will be use to determine the companied (h.p.i.) in (NC-2D: RSP) as:

$$
\begin{aligned}
& a \hat{r}^{2}=a r^{2}-a \theta L_{z} \\
& \frac{b}{\hat{r}^{2}}=\frac{b}{r^{2}}+\frac{\theta b}{r^{4}} L_{z} \\
& \frac{\hat{p}^{2}}{2 m_{0}}=\frac{p^{2}}{2 m_{0}}+\frac{\bar{\theta}}{2 m_{0}} L_{z}
\end{aligned}
$$

From above relations, one can write the deformed operator $V_{\text {h.p.i }}(\hat{r})$ for companied (h.p.i.) and the noncommutative kinetic term $\frac{\hat{p}^{2}}{2 m_{0}}$, respectively: 


$$
\left\{\begin{array}{l}
V_{\text {h.p.i }}(\hat{r})=a \hat{r}^{2}+\frac{b}{\hat{r}^{2}} \\
\frac{\hat{p}^{2}}{2 m_{0}}=-\frac{1}{2 m_{0}}\left(\frac{1}{r} \frac{\partial}{\partial r}\left(r \frac{\partial}{\partial r}\right)+\frac{1}{r^{2}} \frac{\partial^{2}}{\partial \varphi^{2}}\right)+\frac{\bar{\theta}}{2 m_{0}} L_{z}
\end{array}\right.
$$

Which allow us to obtaining the global potential operator $H_{\text {h.p.i }}(\hat{r})$ for combined (h.p.i.) in both (NC-2D RSP) as:

$$
H_{\text {h.p.i }}(\hat{r})=a r^{2}+\frac{b}{r^{2}}+\left(\theta\left(\frac{b}{r^{4}}-a\right)+\frac{\bar{\theta}}{2 m_{0}}\right) L_{z}
$$

It's clearly, that the two first terms are given the ordinary companied (h.p.i.) in 2D space, while the rest terms are proportional's with two infinitesimals parameters ( $\theta$ and $\bar{\theta})$ and then gives the terms of perturbations $H_{\text {h.p.i }}(r)$ in (NC-2D RSP) as:

$$
H_{\text {h.p.i }}(r)=\left(\theta\left(\frac{b}{r^{4}}-a\right)+\frac{\bar{\theta}}{2 m_{0}}\right) L_{z}
$$

This can be writing to the equivalent form:

$$
H_{\text {h.p.i }}(r)=\left(\theta\left(\frac{b}{r^{4}}-a\right)+\frac{\bar{\theta}}{2 m_{0}}\right) \vec{S} \vec{L}
$$

We orient the spin to the $(\mathrm{Oz})$ which appear parallel with $L_{z}$, which allow us to write, the perturbative term $H_{\text {ihopiq } p}(r)$ as follows:

$$
H_{\text {h.p. }}(r)=\left(\theta\left(\frac{b}{r^{4}}-a\right)+\frac{\bar{\theta}}{2 m_{0}}\right)\left(\vec{J}^{2}-\vec{L}^{2}-\vec{S}^{2}\right) .
$$

We have replaced $(\vec{S} \vec{L})$ by $\frac{1}{2}\left(\vec{J}^{2}-\vec{L}^{2}-\vec{S}^{2}\right)$, this operator traduce the coupling between spin and orbital momentum. Now we replace $\Psi(\dot{r})$ by $R_{n l}(r) \Phi(\varphi)$ in eq. (22), after profound straightforward calculation, one can show that, the radial function $R_{n l}(r)$ and the angular function $\Phi(\varphi)$ are satisfied the following two equations, in (NC-2D: RSP), respectively:

$$
\begin{aligned}
& \left(\frac{d^{2}}{d r^{2}}+\frac{1}{r} \frac{d}{d r}-\frac{m^{2}}{r^{2}}-2 m_{0}\left(E_{n c-i h}-a r^{2}-\frac{b}{r^{2}}-\left(\theta\left(\frac{b}{r^{4}}-a\right)+\frac{\bar{\theta}}{2 m_{0}}\right) L_{z}\right)\right) R_{n l}(r)=0 \\
& \frac{d^{2} \Phi_{m}(\varphi)}{d \varphi^{2}}+m^{2} \Phi_{m}(\varphi)=0
\end{aligned}
$$

The set $\left(H_{n 2 s p(\text { h.p.i) }}, \mathrm{J}^{2}, \mathrm{~L}^{2}, \mathrm{~S}^{2}\right.$ and $\left.J_{z}\right)$ forms a complete of conserved physics quantities and the eigen-values of the spin orbital coupling operator are $k_{ \pm} \equiv \frac{1}{2}\left\{\left(l \pm \frac{1}{2}\right)\left(l \pm \frac{1}{2}+1\right)+l(l+1)-\frac{3}{4}\right\}$ corresponding: $j=l+\frac{1}{2}$ (spin up) and $j=l-\frac{1}{2}$ (spin down), respectively, then, one can form a diagonal $(2 \times 2)$ matrix, with non null elements are $\left(H_{n s p(\text { h.p.i })}\right)_{11}$ and $\left(H_{n 2 s p(\text { h.p.i })}\right)_{22}$ for companied Harmonic potential and its inverse in (NC-2D RSP) as:

$$
\begin{aligned}
& \left(H_{n s p(\text { h.p.i. }}\right)_{11}=-\frac{\Delta}{2 m_{0}}+a r^{2}+\frac{b}{r^{2}}+k_{+}\left(\theta\left(\frac{b}{r^{4}}-a\right)+\frac{\bar{\theta}}{2 m_{0}}\right) \\
& \text { if } j=l+\frac{1}{2} \Rightarrow \text { spin up } \\
& \left(H_{n s p(\text { h.p.i. })}\right)_{22}=-\frac{\Delta}{2 m_{0}}+a r^{2}+\frac{b}{r^{2}}-k_{-}\left(\theta\left(\frac{b}{r^{4}}-a\right)+\frac{\bar{\theta}}{2 m_{0}}\right) \\
& \text { if } j=l-\frac{1}{2} \Rightarrow \text { spin down }
\end{aligned}
$$

The energies $E_{\text {n2u(h.p.i) }}$ and $E_{\text {n2d(h.p.i) }}$ of a particle fermionic with spin up and spin down are determined, respectively, for companied Harmonic potential and its inverse in (NC-2D: RSP) as:

$$
\begin{aligned}
& E_{\text {n2u(h.p.i) }}=E_{n l}+E_{\text {u2(h.p.i.) }} \\
& E_{\text {n2d(h.p.i) }}=E_{n l}+E_{\text {d2(h.p.i.) }}
\end{aligned}
$$


Where $E_{\text {u2(h.p.i.) }}$ and $E_{\text {d2(h.p.i.) }}$ are the modifications to the energy levels, associate with spin up and spin down, respectively, at first order of two parameters $\theta$ and $\bar{\theta}$ obtained by applying the perturbation theory, as follows:

$$
\begin{aligned}
& E_{\text {u2(h.p.i.) }}=2 k_{+}\left(\theta T_{\text {s(h.p.i.) }}+\frac{\bar{\theta}}{2 m_{0}} T_{\text {p(h.p.i.) }}\right) \\
& E_{\text {d2(h.p.i.) }}=-2 k_{-}\left(\theta T_{\text {s(h.p.i.) }}+\frac{\bar{\theta}}{2 m_{0}} T_{\text {p(h.p.i.) }}\right)
\end{aligned}
$$

In above two equations $T_{\text {s(h.p.i.) }}$ and $T_{\text {p(h.p.i.) }}$ are given by:

$$
\begin{aligned}
& T_{\text {s(h.p.i.) }}=A \int_{0}^{+\infty}\left[\exp \left(-\frac{\mu}{2} r^{2}\right) r^{k}{ }_{1} F\left(-n, k+1, r^{2}\right)\right]^{2}\left(\frac{b}{r^{3}}-a r\right) d r \\
& T_{\text {p(h.p.i.) }}=A \int_{0}^{+\infty}\left[\exp \left(-\frac{\mu}{2} r^{2}\right) r^{k}{ }_{1} F\left(-n, k+1, r^{2}\right)\right]^{2} d r
\end{aligned}
$$

Where $A=\left(\frac{2 \mu^{(k+1)} n !}{\Gamma(k+n+1)}\right)\left(\frac{(n+k) !}{n ! k !}\right)^{2}$, the first part in eq. (34) can be equal the sum of two terms $T_{\text {s(h.p.i.) }}^{1}$ and $T_{\text {s(h.p.i.) }}^{2}$ as:

$$
\begin{aligned}
& \left.T_{\text {sh.p.i.) }}^{1}=A b \int_{0}^{+\infty} \exp \left(-\mu r^{2}\right) r^{2 k-3 .} \cdot{ }_{1} F_{1}\left(-n, k+1, r^{2}\right)\right]^{2} d r \\
& \left.T_{\text {s(h.p.i.) }}^{2}=-A a \int_{0}^{+\infty} \exp \left(-\mu r^{2}\right) r^{2 k+1} \cdot{ }_{1} F_{1}\left(-n, k+1, r^{2}\right)\right]^{2} d r
\end{aligned}
$$

It's convent to introduce a new variable $v=\mu r^{2}$ and then eq. (33) take the equivalent form:

$$
\begin{aligned}
& T_{\mathrm{s}(\mathrm{h} . \mathrm{p} \text {.i. })}^{1}=\frac{A b}{2 \mu^{k-1}} \int_{0}^{+\infty} v^{k-2} \exp (-v)\left[F_{1}\left(-n, k+1, \frac{v}{\mu}\right)\right]^{2} d v \\
& T_{\text {s(h.p.i.) }}^{2}=-\frac{a}{2 \mu^{k+2}} \int_{0}^{+\infty} v^{k+1} \exp (-v)\left[F_{1}\left(-n, k+1, \frac{v}{\mu}\right)\right]^{2} d v
\end{aligned}
$$

Applying the following special integration [38]:

$$
\begin{aligned}
& \int_{0}^{+\infty} v^{\alpha-1 .} \exp (-v)\left[{ }_{1} F_{1}(-n, \gamma, v)\right]^{2} d v= \\
& \frac{n ! \Gamma(\alpha)}{\gamma(\gamma+1) . .(\gamma+n-1)}\left(\begin{array}{c}
1+\frac{n(\gamma-\alpha-1)(\gamma-\alpha)}{1^{2} \gamma}+ \\
+\frac{n(n-1)(\gamma-\alpha-2)(\gamma-\alpha-1)(\gamma-\alpha)(\gamma-\alpha+1)}{1^{2} 2^{2} \gamma(\gamma+1)}+.
\end{array}\right)
\end{aligned}
$$

Then, we can prove that first integral in equation (33) is:

$$
\begin{aligned}
& \int_{0}^{+\infty} \exp (-v) v^{\cdot k-2}\left[{ }_{1} F_{1}\left(-n, k+1, \frac{v}{\mu}\right)\right]^{2} d v= \\
& =B\left(1+\frac{n(k+1-(k-1)-1)(k+1-(k-1))}{k+1}+\ldots\right)
\end{aligned}
$$

Where $B=\frac{n !(k-1)}{(k+1)(k+2)(k+n)}$, the eq. (35) can be simplified to the form:

$$
\int_{0}^{+\infty} \exp (-v) v^{k-2}\left[{ }_{1} F_{1}\left(-n, k+1, \frac{v}{\mu}\right)\right]^{2} d v=\frac{K(2 n+k+1)}{k+1}
$$

And the second integral in equation (36) can be written as follow:

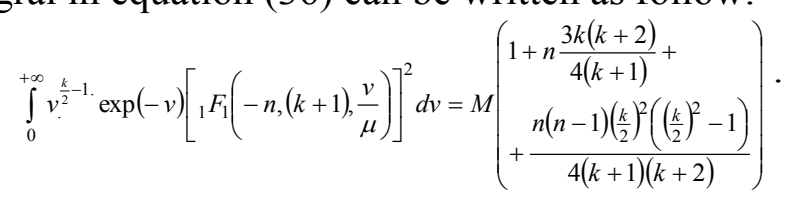

Where $M=\frac{\Gamma\left(\frac{k}{2}\right)}{(k+1)(k+2)(k+n)}$, which allow us to get $T_{\text {s(h.p.i.) }}^{1}$ as follows: 


$$
T_{\text {s(h.p.i.) }}^{1}=\frac{L_{(\text {(h.p.i.) }}(n, k)}{(k+1)}
$$

The obtained factor: $L_{(\text {(h.p.i.) }}(n, k)$ winches represent the roll of (NC-2D) space as:

$$
L_{\text {(h.p.i.) }}(n, k)=\frac{A b B(2 n+k+1)}{2 \mu^{k-1}}-\frac{A a B}{2 \mu^{k+2}}\left(\frac{3 n k(k+2)+4(k+1)}{4}+\frac{n(n-1)\left(\frac{k}{2}\right)^{2}\left(\left(\frac{k}{2}\right)^{2}-1\right)}{4(k+2)}\right) .
$$

Now, if we replace ${ }_{1} F_{1}\left(-n, k+1, r^{2}\right)$ by its values $\frac{n ! k !}{(k+n) !} L_{n}^{k}\left(r^{2}\right)$, the second part of the equation (34), can be written as:

$$
T_{\text {p(h.p.i. }}=A\left(\frac{n ! k !}{(k+n) !}\right)^{2} \int_{0}^{+\infty} \exp \left(-\mu r^{2}\right) r^{2 k}\left[L_{n}^{k}\left(r^{2}\right)\right]^{2} d r
$$

It's necessary to introduce a new variable $r^{2}=x$ and then we rewriting $T_{\text {p(h.p.i.) }}$ as have:

$$
T_{\text {p(h.p.i. })}=\frac{A}{2}\left(\frac{n ! k !}{(k+n) !}\right)^{2} \int_{0}^{+\infty} \exp (-\mu x) x^{k-\frac{1}{2}}\left[L_{n}^{k}(x)\right]^{2} d x .
$$

Now, we apply the integral $[7,38]$ :

$$
\int_{0}^{+\infty} x^{A} \cdot \exp (-x)\left[L_{B}^{A}(x)\right]^{2} d x=\frac{\Gamma(A+B+1)}{B !}
$$

Which allow us to writing (NC-2D) phase contribution $T_{\text {ph.p.i.) }}$ as:

$$
T_{\text {p(h.p.i.) }}=\frac{A n !}{2}\left(\frac{k !}{(k+n) !}\right)^{2} \Gamma\left(k-\frac{1}{2}+n+1\right)
$$

The modification to the energy levels, associate with spin up and spin down, at first order of $\theta$ and $\bar{\theta}$ for companied Harmonic potential and its inverse in both (NC-2D: RSP) are given by:

$$
\begin{aligned}
& E_{\text {u2(h.p.j.) }}=A k_{+}\left(\theta \frac{L_{(\mathrm{hpip})}(n, k)}{k+1}+\frac{\bar{\theta} n !}{2 m_{0}}\left(\frac{k !}{(k+n) !}\right)^{2} \Gamma\left(k-\frac{1}{2}+n+1\right)\right) \\
& E_{\text {d2 (h.p.i. })}=-A k_{-}\left(\theta \frac{L_{(\text {h.p.i) }}(n, k)}{k+1}-\frac{\bar{\theta} n !}{2 m_{0}}\left(\frac{k !}{(k+n) !}\right)^{2} \Gamma\left(k-\frac{1}{2}+n+1\right)\right)
\end{aligned}
$$

The first two terms in above two equations are clearly determine the physics contributions of (NC2D) space, while the second parts are gives physics contributions of (NC-2D) phase, we conclude, from Eqs. (47) and (32) one can resume the total energy of electron with two polarisations spin up and down $E_{\text {nu(h.p.i.) }}$ and $E_{\text {nd(h.p.i.) }}$ corresponding $\left(H_{\text {(h.p.i.) }}\right)_{11}$ and $\left(H_{\text {(h.p.i.) }}\right)_{22}$, respectively for companied Harmonic potential and its inverse in (NC-2D: RSP) as:

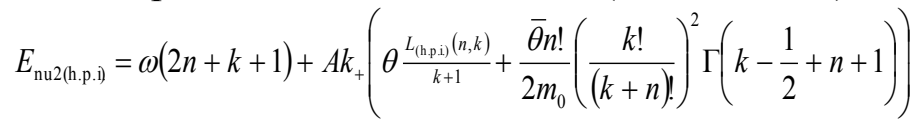

$$
\begin{aligned}
& E_{\text {nd2h.p.i. })}=\omega(2 n+k+1)-A k_{-}\left(\theta \frac{L_{(\ln +\hat{k})}(n, k)}{k+1}-\frac{\bar{\theta} n !}{2 m_{0}}\left(\frac{k !}{(k+n) !}\right)^{2} \Gamma\left(k-\frac{1}{2}+n+1\right)\right)
\end{aligned}
$$

Thus, the eigenvalues of energies are real's and then the NC Hamiltonian is Hermitian for companied Harmonic potential and its inverse, in another hand it's possible to writing it as follows:

$$
H_{N C \text {-(h.p.i.) }}=H_{\text {(h.p.i.) }}+H_{s o-\text { (h.p.i.) }}
$$

Where $H_{\text {(h.p.i.) }}$ and $H_{s o-(\text { (h.p.i.) }}$ are determined from, the following relation, respectively:

$$
\begin{aligned}
& H_{\text {(h.p.i.) }}=\left(-\frac{1}{2 m_{0}}\left(\frac{1}{r} \frac{\partial}{\partial r}\left(r \frac{\partial}{\partial r}\right)+\frac{1}{r^{2}} \frac{\partial^{2}}{\partial \varphi^{2}}\right)+a r^{2}+\frac{b}{r^{2}}\right)\left(\begin{array}{ll}
1 & 0 \\
0 & 1
\end{array}\right) \\
& H_{s o-\text { (h.p.i.) }}=\left(\theta\left(\frac{b}{r^{4}}-a\right)+\frac{\bar{\theta}}{2 m_{0}}\right)\left(\begin{array}{cc}
k_{+} & 0 \\
0 & -k_{-}
\end{array}\right)
\end{aligned}
$$


Here $H_{\text {(h.p.i.) }}$ represent an electron interacted exactly with companied Harmonic potential and its inverse in commutative $2 \mathrm{D}$ space while the matrix $H_{s o \text {-(h.p.i.) }}$ denotes to the spin-orbital interaction. Furthermore, if we apply the three-following steps:

$$
\begin{aligned}
& \left(\theta\left(\frac{b}{r^{4}}-a\right)+\frac{\bar{\theta}}{2 m_{0}}\right) L_{z} \rightarrow\left(\chi\left(\frac{b}{r^{4}}-a\right)+\frac{\bar{\sigma}}{2 m_{0}}\right) \vec{B} \vec{L} \\
& \theta \rightarrow \chi B \\
& \bar{\theta} \rightarrow \bar{\sigma} B
\end{aligned}
$$

In addition to these steps, we choose the magnetic field $\overleftrightarrow{B}=B \overleftrightarrow{k}$, here $\chi$ and $\bar{\sigma}$ are infinitesimal real proportional's constants, the magnetic moment $\overleftrightarrow{\mu} \equiv 1 / 2$ and $(-\overleftrightarrow{S} \overleftrightarrow{B})$ denote to the ordinary Hamiltonian of Zeeman Effect, we obtains the modified new Hamiltonian for companied Harmonic potential and its inverse in (NC-2D: RSP) as:

$$
H_{m-\text { (h.p.i.) }}=\left(\chi\left(\frac{b}{r^{4}}-a\right)+\frac{\bar{\sigma}}{2 m_{0}}\right)(\vec{B} \vec{J}-\overleftrightarrow{S} \overleftrightarrow{B})
$$

The above operator represents two fundamentals interactions: the first one between spin and external uniform magnetic field (ordinary Zeeman Effect) while the other is a new coupling between the momentum of electron and external uniform magnetic field. As it's mentioned previously, the two parameters $\theta$ and $\bar{\theta}$ was played an opposite rolls which allow us to introduce the following gauge fixing condition of Maireche:

$$
\bar{\theta}=-\rho \theta
$$

Here $\rho$ is a positive constant, our obtained spectrum, will be proportioned only to one infinitesimal parameter $\theta$ and positive constant.

\section{RESULTS AND CONCLUSIONS}

In present work, we solved the Schrödinger equation with the companied Harmonic oscillator

\begin{tabular}{|c|c|c|}
\hline The total energy in 2D & NC space-spin orbit & NC phase-spin Orbit \\
\hline \multirow{2}{*}{$\begin{array}{l}E_{n l}=\sqrt{\frac{8 \hbar^{2}}{M}}\left(n+\frac{1}{2}+\frac{1}{4}\left(\begin{array}{l}4 m^{2} \\
+\frac{8 M b}{\hbar^{2}}\end{array}\right)^{\frac{1}{2}}\right) \\
=\omega(2 n+k+1)\end{array}$} & $\begin{array}{l}E_{n s-(\text { h.p.i.) }}= \\
\theta A k_{+} \frac{L_{(\text {h.p.i.) }}(n, k)}{k+1} \text { for spin up }\end{array}$ & $\begin{array}{l}E_{n p-(\text { h.p.i.) }}=\frac{\bar{\theta} n ! A k_{+}}{2 m_{0}} \\
\left(\frac{k !}{(k+n) !}\right)^{2} \Gamma\left(k-\frac{1}{2}+n+1\right) \\
\text { for spin down }\end{array}$ \\
\hline & $\begin{array}{l}E_{n s-(\mathrm{h} . \mathrm{p} . \mathrm{i} .)}= \\
-A k_{-} \theta \frac{L_{(\mathrm{h} . \mathrm{p} .)}(n, k)}{k+1} \\
\text { for spin down }\end{array}$ & $\begin{array}{l}E_{n p-s o d}=\frac{\bar{\theta} n ! A k}{2 m_{-}} \\
\left(\frac{k !}{(k+n) !}\right)^{2}\left[\left(k-\frac{1}{2}+n+1\right)\right. \\
\text { for spin down }\end{array}$ \\
\hline
\end{tabular}
potential and its inverse in both noncommutativity two dimensional of real space and phase, we used both Boopp's shift method and standard perturbation theory to obtain the following resumed structure of the energy levels in a new symmetric, in two tables:

Table: 1 
Table: 2

\begin{tabular}{|c|c|c|}
\hline NC space-magnetic & NC phase- magnetic & $\begin{array}{c}\text { The spectrum energy in (NC-2D) } \\
\text { phase and space }\end{array}$ \\
\hline \multirow[b]{2}{*}{$E_{n p-m}=\theta A m \frac{L(n, k)}{k+1}$} & \multirow{2}{*}{$\begin{array}{l}E_{n p-m}=\frac{\bar{\theta} A n ! m}{2 m_{0}} \\
\left(\frac{k !}{(k+n) !}\right)^{2} \Gamma\left(k-\frac{1}{2}+n+1\right)\end{array}$} & $\begin{array}{l}E_{\text {gnul(t.p.p) }}=E_{n l}+E_{n s-s o u}+\text { for spin down } \\
+E_{n p-s o u}+E_{n s-m}+E_{n p-m}\end{array}$ \\
\hline & & $\begin{array}{l}E_{\text {gndh.p.i. }}=E_{n l}+E_{n s-s o d}+\text { for spin down } \\
+E_{n p-s o d}+E_{n s-m}+E_{n p-m}\end{array}$ \\
\hline
\end{tabular}

Thus, the obtained global full spectrum for companied $E_{\text {gnu(h.p.i) }}$ (for spin up) and $E_{\text {gndh.p.i.), }}$ (for spin down) for Harmonic oscillator potential and its inverse:

$$
\begin{aligned}
& E_{\text {gnu(h.p.j) }}=\omega(2 n+k+1)+A \theta\left(\begin{array}{l}
\frac{L_{\left(\frac{h p i}{2}(n), k\right)}}{k+1}- \\
-\frac{\rho n !}{2 m_{0}}\left(\frac{k !}{(k+n) !}\right)^{2} \Gamma\left(\frac{2(k+n)+1}{2}\right)
\end{array}\right)\left(k_{+}+m\right) \\
& \text { : for-spin-up } \\
& E_{\text {gndh.p.i. })}=\omega(2 n+k+1)+A \theta\left(\begin{array}{l}
\frac{L_{(\text {h.pi }}(n, k)}{k+1}+ \\
-\frac{\rho n !}{2 m_{0}}\left(\frac{k !}{(k+n) !}\right)^{2} \Gamma\left(\frac{2(k+n)+1}{2}\right)
\end{array}\right)(k+m) \\
& \text { : for-spin-down }
\end{aligned}
$$

And the corresponding formed noncommutative diagonal Hamiltonian for studied potential in (NC2D: RSP) with elements $\left(H_{n c(\text { h.p.i. })}\right)_{11},\left(H_{n c(\text { h.p.i. })}\right)_{22},\left(H_{n c(\text { h.p.i. })}\right)_{12}$ and $\left(H_{n c(\text { h.p.i. })}\right)_{21}$ :

$$
\begin{aligned}
& \left(H_{n c(\text { h.p.i. })}\right)_{11}=\left(-\frac{\Delta}{2 m_{0}}+a r^{2}+\frac{b}{r^{2}}\right)+\left(\theta\left(\frac{b}{r^{4}}-a\right)+\frac{\bar{\theta}}{2 m_{0}}\right) k_{-}+\left(\chi\left(\frac{b}{r^{4}}-a\right)+\frac{\bar{\sigma}}{2 m_{0}}\right) m \\
& \left(H_{n c(\text { h.p. p. . ) }}\right)_{22}=\left(-\frac{\Delta}{2 m_{0}}+a r^{2}+\frac{b}{r^{2}}\right)+\left(\theta\left(\frac{b}{r^{4}}-a\right)+\frac{\bar{\theta}}{2 m_{0}}\right) k_{-}+\left(\chi\left(\frac{b}{r^{4}}-a\right)+\frac{\bar{\sigma}}{2 m_{0}}\right) m^{-} \\
& \left(H_{n c(\text { h.p.i. }))_{12}}=\left(H_{n(\text { h. }} . \text {.i. }\right)\right)_{21}=0
\end{aligned}
$$

Which allow us to obtaining the effect of the operators $\left(H_{n c(\text { h.p.i. })}\right)_{11}$ and $\left(H_{n c(\text { h.p.i. })}\right)_{22}$ on the complex wave function $\Psi(r, \varphi)$ as:

$$
\left\{\begin{array}{l}
\left(H_{n c(\text { h.p.i.) }}\right)_{11} \Psi(r, \varphi)=E_{\text {gnu(h.p.l) }} \Psi(r, \varphi) \\
\left(H_{n c(\text { (h.p.i.) }}\right)_{22} \Psi(r, \varphi)=E_{\text {gnd(h.p.i) }} \Psi(r, \varphi)
\end{array}\right.
$$

Thus, we can deduce that, the new found symmetries capable of describing many fields like the modified Zeeman Effect, spin orbital interaction and some other applications in different types of materials science.

\section{ACKNOWLEGMENTSA}

This work was supported with search laboratory of: Physique et Chimie des matériaux, in university of M'sila, Algeria.

\section{References}

[1] H. Hassanabadi, et al, International Journal of the Physical Sciences, 2011, 6 (3), 583.

[2] S. K. Bose, IL NUOVO CIMENTO, 1994, 109, 1217.

[3] L. Buragohai and S. A. Ahmed, Lat. Am. J. Educ, 2009, 3, 573.

[4] S. K. Bose, IL NUOVO CIMENTO, 1994, 109B, N. 3, 311.

[5] I. Sameer and S. Ramazan, CEJP.2007, 5, 4, 516. 
[6] E. S. Santos and G. R. Melo, IJP, 2011, 50, 332.

[7] Tapas Das, Subhankar Ray and Altug Arda, Exact Solution of N-dimensional Radial Schrödinger Equation with Pseudoharmonic Potential via Laplace Transforme Approach. arXiv: $1308.5295 \mathrm{v} 1$ (math-ph) (2013).

[8] A. E. F. Djema and H. Smail. Commun, Theor. Phys, 2004, 41, 837.

[9] Shi-Hai and S. Guohuo, Foundation of Physics Letters, 2003, 16, 357.

[10] Shi-Hai D, Int J Theor Phys,2000, 39, 4, 1119-1128.

[11] Shi-Hai, D., Foundations of physics Letters,2002, 15, 4, 385-395.

[12] Shi-Ha,i D, Int J Theor Phys,2001, 40, 2, 559-567.

[13] Shi-Hai, D.; Guo-Huo, S, Foundation of Physics Letters, 2003, 16, 357-367.

[14] L. Buragohain and, S. A.S. Ahmed, Lat. Am. J. Phys. Educ, 2010, 4, 1.

[15] Anjana, Sinha, Int J Theor Phys,1998, 37, 7, 2055-2065.

[16] Akpan N., Eno J. Ibango, Oladunjoye A. Awoga, Louis E. Akpabio and Akaninyene D. Antia. Journal of Modern Physics, 2012 3, 1849-1855.

[17] O.M. Al-dossary.International journal of quantum Chemistry, 2006, Vol 107, No 10, 547.

[18] C. B. Compean and M. Kirchbach, Journal of physics A, 2007, Vol 39, No 3, pp 2040-2046.

[19] A. Connes. Noncommutative geometry, 1st ed. (Academic Press, Paris, France, 1994).

[20] H. Snyder, Phys. Rev, 1947, 71, 38-41.

[21] Sergio, D., Journal of Physics, 2006, 53, 793.

[22] R. Szabo, Phys. Rep, 2003, 378, 207.

[23] D. T. Jacobus. PhD, (Department of Physics, Stellenbosch University, South Africa, 2010).

[24] N. G. Deshpande, PRAMAN journal of physics, 2003, 60, 189.

[25] M. R. Vilela. Physics Lettres, 210, 232 (1996).

[26] B. Miraza and M. Mohadesi, Commun. Theor. Phys, 2004, 42, 664.

[27] Y. Yuan, K. Li, J. Wang and C. Chen. Chinese Physics C, 2010, 34(5): 543.

[28] J. Wang, K. Li and s. Dulat, Chinese Physics C, 2008, 10, 803.

[29] Abdelmadjid Maireche, Life Sci. J. 2014,11(6), 353.

[30] Abdelmadjid Maireche, The African Rev. Physics. 2014, 9:0060, 479.

[31] Abdelmadjid Maireche. J. Nano- Electron. Phys, 2015, 7 No 2, 02003.

[32] Abdelmadjid Maireche, The African Rev. Physics, 2014, 9:0025.

[33] Zaim Slimane, Int J Theor Phys, 2014, 53, 2014-2023.

[34] A.E.F. Djemei and H. Smail. Commun, Theor. Phys. (Beijinig, China), 2004, 41 pp.837-844.

[35] J. Gamboa, M. Loewe and J. C. Rojas. Non- Commutative Quantum Mechanics. arXiv:hepth/0010220v4. (2001).

[36] L. Mezincescu, Star product in quantum mechanics.arXiv:hep-th/0007046v2. (2000).

[37] Mirza B, Zarei M., Eur. Phys. J. C, 2004, 32: 583.

[38] I. S. Gradshteyn and I. M. Ryzhik, Table of Integrals, Series and Products, (7th. ed.; Elsevier, USA 2007). 